\title{
La Renaissance africaine, des idées à la pierre
}

L'infrastructure de Cheikh Anta Diop, la culture de Léopold Sédar Senghor et la sculpture d'Abdoulaye Wade

\section{Martin Mourre}

\section{(2) OpenEdition}

1 Journals

\section{Édition électronique}

URL : http://journals.openedition.org/etudesafricaines/20878

DOI : 10.4000/etudesafricaines. 20878

ISSN : $1777-5353$

Éditeur

Éditions de l'EHESS

\section{Édition imprimée}

Date de publication : 1 septembre 2017

Pagination : 719-750

ISBN : 978-2-7132-2686-1

ISSN : 0008-0055

Référence électronique

Martin Mourre, «La Renaissance africaine, des idées à la pierre », Cahiers d'études africaines [En ligne], 227 | 2017, mis en ligne le 01 septembre 2019, consulté le 01 janvier 2020. URL : http://

journals.openedition.org/etudesafricaines/20878; DOI : 10.4000/etudesafricaines.20878 


\title{
La Renaissance africaine, des idées à la pierre
}

\author{
L'infrastructure de Cheikh Anta Diop, \\ la culture de Léopold Sédar Senghor \\ et la sculpture d'Abdoulaye Wade
}

«Plus haut que la Statue de la Liberté» : c'est ainsi que de nombreux commentateurs ont qualifié le Monument de la Renaissance, ce gigantesque ensemble statuaire de bronze et de cuivre, haut de cinquante-trois mètres, au moment de son inauguration sur la presque-île du Cap-Vert, le 3 avril 2010 à la veille des fêtes du cinquantenaire de l'indépendance du Sénégal ${ }^{1}$. En 2010, l'édification de cette statue fut vivement dénoncée. Elle provoqua l'ire des imams qui s'indignèrent de la tenue jugée trop courte du personnage féminin ainsi que celle d'intellectuelles sénégalaises féministes qui soulignèrent que le personnage féminin était relégué au second plan. Des polémiques surgirent quant à la paternité du monument ${ }^{2}$. On dénonça son coût et on s'indigna du fait que le président Wade s'octroie $35 \%$ des bénéfices, au titre de la propriété intellectuelle, sur les futurs droits d'entrée (De Jong \& Foucher $2010: 190$ ). Mais pour les partisans du président, cette sculpture était censée symboliser « le combat du mouvement noir, porté par des millions de Noirs dans le monde, et qui fait du Sénégal, un lieu de mémoire de la renaissance et de la résistance africaines $»^{3}$. Si certains ont dénoncé une tentative de «monumentalisation du passé » par le président Wade (Dramé 2011), cette statue fut auréolée, en 2010, de tout un ensemble de discours, de la part d'une opposition hétéroclite

1. Je tiens à remercier les deux évaluateurs anonymes pour les remarques qu'ils ont faites d'une précédente version de cet article.

2. C'est d'abord le sculpteur sénégalais Ousmane Sow qui fut pressenti pour réaliser le monument, avant que sa réalisation ne revienne à l'architecte et conseiller du président Wade, Pierre Goudiaby. La mise en œuvre fut exécutée par l'entreprise nord-coréenne Mansudae Overseas Projects.

3. Non signé, «Le Monument de la Renaissance africaine, une priorité stratégique majeure (conseiller) », Agence de presse sénégalaise, 14 janvier 2010. 
mais aussi du pouvoir sénégalais, donnant ainsi à voir « un écran sur lequel différents segments de la population projettent leurs préoccupations politiques et morales contradictoires » (De Jong \& Foucher 2010 : 188). Ce monument s'apparente ainsi à une " forme d'énonciation d'une identité panafricaine » (ibid. : 194) et c'est la formulation de cette identité, dans une moyenne durée historique, qui intéresse ici. Il convient donc de prendre en charge la consistance de cet imaginaire contemporain, non seulement dans la mise en scène liée à son inauguration mais aussi dans l'appellation du monument qui semble exprimer une nouvelle vision politique du panafricanisme en lien avec des débats passés.

Le panafricanisme, cet objet politique encore mal identifié, a une longue histoire (Boukary-Yabara 2014). Il importe d'en faire la généalogie au Sénégal en s'attachant à retracer certains débats économiques, liés à l' " infrastructure », des débats quant à la définition d'une identité africaine " culturelle», des débats, enfin, quant à la gouvernance des symboles, liés alors ici à la sculpture. Ces trois axes seront abordés de manière chronologique, bien qu'ils se superposent. Ils seront retracés à travers les héritages intellectuels sénégalais du président Wade, afin d'illustrer comment la « Renaissance africaine » incarnée dans cet ensemble statutaire, est devenue un tel signifiant flottant. Deux intellectuels, Cheikh Anta Diop et Léopold Sédar Senghor, parmi les auteurs les plus féconds au $\mathrm{XX}^{\mathrm{e}}$ siècle, ont longuement abordé ces thèmes, en théorisant ou en s'inspirant d'une idée de la Renaissance. Ces deux intellectuels, Abdoulaye Wade les connaît bien. Sans entreprendre une comparaison entre la pensée de Diop et Senghor - les limites d'un tel article ne le permettent pas et l'exercice a déjà été partiellement entrepris (Diop 2006; Tine 2005) — on peut suivre, à travers les itinéraires croisés de ces deux militants et responsables politiques, le legs qu'ils ont laissé dans la pensée et la pratique politique sénégalaise. Ce legs s'est matérialisé dans le projet « monumental » d'Abdoulaye Wade. Dès 2007, lors des commémorations de la fête nationale sénégalaise, l'hymne national fut remplacé par celui de la « Renaissance africaine », dont l'auteur n'est autre qu'Abdoulaye Wade lui-même. Il s'agit sûrement d'une des premières séquences où "Gorgui» — surnom attribué à Wade et que l'on pourrait traduire par «le vieux » — arrivé au pouvoir en 2000, mobilise publiquement le concept de «renaissance » pour en faire un ferment d'unité nationale mais aussi pour asseoir sa stature internationale. Chanté en français et en wolof, les paroles de cet hymne sont tirées de l'ouvrage Un destin pour l'Afrique que Wade, alors opposant au pouvoir d'Abdou Diouf, publie en 1989, où le chant figure toutefois sous le titre d' «Hymne pour l'Afrique». Dans cet ouvrage, Abdoulaye Wade y développe des thèses qu'il emprunte notamment à Kwame Nkurumah, le premier président ghanéen, considéré 
comme le père africain du panafricanisme, ou tout du moins comme son plus célèbre porte-parole. Diop et Senghor sont également cités.

Moins que d'entreprendre une analyse de la pensée politique du président Wade, cet article revient d'abord sur des débats entre Diop et Senghor, notamment dans leurs premiers écrits, qui illustrent deux conceptions de la «Renaissance africaine ». Cela nous permet, ensuite, de mieux comprendre la décision prise en 2003 d'ériger ce Monument de la Renaissance de plus de 22000 tonnes $^{4}$, qui semble ainsi cristalliser ces différents rapports au passé, et ces différentes visions du progrès pour l'Afrique, énoncés par Léopold Sédar Senghor et Cheikh Anta Diop.

La mémoire historique, telle que définie par Maurice Halbwachs (1997 [1950]) renvoie à la construction par un groupe d'une représentation consciente et volontaire de son passé, opposée, ou plutôt mise en miroir, avec une mémoire vive, c'est-à-dire des souvenirs portés plus directement par des individus. Si l'un des points d'entrée actuels de cette réflexion d'Halbwachs sur ce qui forme la « mémoire collective » d'un groupe peut être celui stricto sensu des politiques mémorielles, on doit également l'aborder au Sénégal à travers une histoire de différents concepts - liés progressivement, et de plus en plus, à différentes constructions statuaires - qui ont rythmé la politique du pays sur plus d'un demi-siècle. Abdoulaye Wade apparaît comme un témoin privilégié de cette époque qui commence après la Seconde Guerre mondiale et qui, pendant plusieurs décennies, voit s'affronter politiquement Diop et Senghor. L'œuvre intellectuelle de Wade, et bien qu'on le surnomme parfois «l'Africain le plus diplômé du Caire au Cap », peut difficilement être comparée à celle du pharaon du Kajoor et à celle du poète sérère. Si la critique intellectuelle des deux premiers penseurs a déjà été menée par des auteurs sur plusieurs continents ${ }^{5}$, il s'agit dans cet article de s'appuyer sur une question transversale qui se posait dans le Sénégal des années 2000, celle de la mémoire historique du continent. Senghor, Diop et Wade sont des enfants de la colonisation, et réfléchir sur leurs rapports au passé incite à penser de manière située la production de ces expériences politiques générationnelles, entre la fin des années 1940 et le début des années 1980 pour les deux premiers, en ce début de $\mathrm{XXI}^{\mathrm{e}}$ — dans sa pratique du pouvoir — pour le second.

4. Non signé, «L'ouvrage pèse 22000 tonnes, selon Pierre Goudiaby Atépa », Agence de presse sénégalaise, 3 avril 2010. Toutes les références des articles de l'Agence de presse sénégalaise se trouvent en ligne sur le site de l'APS : $<\mathrm{http} / / /$ archives.aps.sn/article/668 37?lightbox $\% 5$ Bwidth $\% 5 \mathrm{D}=75 \mathrm{p} \&$ lightbox $\% 5$ Bheight $\% 5 \mathrm{D}=90 \mathrm{p}>$.

5. Voir la bibliographie en fin d'article. Nous avons repris certains des ouvrages les plus « classiques » de Cheikh Anta Diop et de Léopold Sédar Senghor, ainsi que des ouvrages critiques qui leur ont été consacré, mais établir une bibliographie exhaustive, autant de leur œuvre que des travaux qui les analysent, excède les limites de cet article. 
Cette « histoire de la mémoire » (Assmann 2003) du continent, de la mémoire historique du continent et sa texture contemporaine, aide à saisir comment le passé, aujourd'hui en Afrique, peut être politisé (Fouéré 2010). Si l'on a souligné comment certains épisodes historiques ont pu s'incarner dans des espaces urbains en Afrique de l'Ouest, notamment au Mali (Arnoldi 2003 ; De Jorio 2006) ou au Sénégal (Diouf 1992 ; De Jong 2008), on s'est en revanche peu intéressé aux liens entre une idée, un concept et un objet.

Le cas de la «Renaissance africaine » semble ainsi paradigmatique. Il illustre, plus généralement, comment le politique s'appuie nécessairement, de manière plus ou moins explicite, sur du passé. Ainsi, le président Wade n'a pas attribué publiquement la paternité du terme «Renaissance » à Cheikh Anta Diop. C'est pourtant bien de lui qu'il a repris cette idée ${ }^{6}$. S'intéresser à cette expression de la «Renaissance africaine » permet de saisir comment des concepts politiques peuvent circuler, s'altérer, prendre des directions inverses de leur sens initial. La notion de « Renaissance », à diverses échelles, est largement présente dans l'espace anglophone du continent, notamment à partir de l'Afrique du Sud post-Apartheid (Crouzel 2000). Elle l'est moins dans l'espace ouest-africain francophone, de sorte que faire la généalogie de ce terme dans cet espace permet d'écrire une autre histoire des débats panafricanistes, dont la première question est celle de la définition même du terme Afrique ${ }^{7}$.

Ces débats prirent une consistance nouvelle parmi l'intelligentsia en formation à Paris durant les années qui suivirent la Seconde Guerre mondiale, où se côtoyaient Diop et Wade, sous le regard de Senghor, leur compatriote et leur aîné d'une génération. Pendant les années 1930, ce dernier, avec d'autres dans ces mêmes espaces parisiens, avait déjà élaboré le concept de " négritude » pour définir le monde noir, des mondes noirs. Cette définition était culturelle et identitaire plus que strictement politique. Une brève confrontation entre Diop et Senghor, quant au patrimoine scientifique qu'ils avaient laissé, alla assurément à l'avantage du premier ${ }^{8}$.

L'un des plus grands romanciers contemporains, le Sénégalais Boris Boubacar Diop, a dressé la comparaison suivante entre les deux hommes en

6. La rédaction du site xibar.net consacre un article à ce sujet : « Seconde mort de Cheikh Anta Diop : Wade décidé à récupérer le concept de "la renaissance africaine" ", 10 février 2010, $<$ http://www.xibar.net/Seconde-mort-de-Cheikh-Anta-Diop-Wade-decide-a-recuperer-leconcept-de-la-renaissance-africaine_a21722.htm>, consulté le 15 janvier 2017.

7. Voir la thèse en cours d'écriture de $\mathrm{S}$. Nakao, Définir l'« Afrique » entre Panafricanisme et Nationalisme en Afrique de l'Ouest. Analyses à travers les transformations sociales au Sénégal, en Haute-Volta et au Ghana, au temps de la décolonisation (1945-1962), (titre provisoire), Paris, EHESS ; Dakar, Université Cheikh Anta Diop.

8. Cheikh Anta Diop a écrit près d'une dizaine d'ouvrages. Comme l'écrit le linguiste et historien sénégalais Pathé DiAGNe (1997 : 18) : «Il n'est personne aujourd'hui, qui, d'une 
2006, alors qu'on fêtait les vingt ans de la mort de Diop et le centenaire de la naissance de Senghor : " En fin de compte, on peut dire que si Senghor a des admirateurs - le grand poète les mérite largement — Cheikh Anta Diop, lui, a des disciples [...]. Senghor est un nom. Cheikh Anta Diop renvoie à une œuvre colossale et féconde » (Diop 2006 : 13). Pour autant au Sénégal, Cheikh Anta Diop est aussi le nom de l'université dakaroise, et celui de la grande avenue qui la longe. Le nom de Senghor, quant à lui, sert à désigner l'aéroport de Dakar et le grand stade de football. Les toponymes urbains représentent alors ces enveloppes charnelles de la mémoire historique, parfois de simples peaux mortes ${ }^{9}$. Ils nous informent sur la répartition de certains héritages de la nation sénégalaise. Une statue représentant Senghor, d'environ deux mètres de haut, a été érigée à Dakar en 2014. Assis, le poète fait face de manière sereine à sa maison sur la corniche et tourne le dos à l'océan. Cette sculpture, dans ses dimensions et son expression, est l'exacte opposée de la «statue de Wade», comme les Dakarois surnomment parfois le Monument de la Renaissance. Cet article, en s'intéressant d'abord à une histoire intellectuelle du terme «Renaissance» durant plus de six décennies au Sénégal, en analysant ensuite la mémoire en tant qu'usage politique, instrumental, du passé, permet d'éclairer un pan de l'imaginaire national (Anderson 1996), tout en précisant, justement, ce rapport entre la communauté politique réelle ${ }^{10}$ et la part d'utopie qui l'institue (Castoriadis 1975).

La première partie de cet article revient sur la relation de Diop et Senghor à la problématique marxiste. Au lendemain de la Seconde Guerre mondiale, il s'agit du paradigme dominant dans la vie politique ouest-africaine, bien qu'il s'élabore également au sein des diasporas africaines. Nous nous concentrons sur leur pensée philosophique, historique et ethnologique. S'intéresser au combat politique que mènent les deux hommes, à travers leurs écrits, incite alors à une réflexion sur la définition de la notion de culture; une culture qui est pensée en relation avec une certaine représentation du passé du continent. C'est aussi le moment où Wade soutient une thèse en économie et où il tente d'élaborer une pensée originale se retrouvant dans le programme qu'il lance

manière ou d'une autre, n'ait été sensibilisé au discours de Cheikh Anta Diop sur l'Égypte négro-pharaonique, la bombe atomique sud-africaine et l'apartheid, la reconquête du désert, le poids des richesses hydroélectriques ou minières de l'Afrique Centrale dans le devenir de tous les peuples du Continent. »

9. On peut faire cette réflexion à travers les noms de rue du centre-ville dakarois renvoyant à des personnalités plus ou moins illustres de la III ${ }^{e}$ République française et dont beaucoup n'évoquent pas grand-chose pour les occupants des lieux. Régulièrement, dans la presse nationale, surgissent d'ailleurs des articles sur la nécessité de débaptiser ces lieux.

10. Pour une lecture critique de la notion de B. Anderson, voir le propos de C. Chivallon (2007). 
à la fin des années 1990 et qui lui permettra d'être élu en 2000. Il s'agit de suivre les filiations entre Wade et ses deux compatriotes. Ces questions, autant chez Diop que chez Senghor, renvoient à la formulation d'une identité collective ; elles sont souvent abordées par le biais d'une approche essentialiste et stratégique. C'est sur le second terme, celui de la stratégie, que nous nous concentrons. Dans une seconde partie, notre propos concerne le rapport à l'histoire, le thème ayant fait la renommée de Cheikh Anta Diop. Si ce dernier élabore sa fameuse thèse sur l'antériorité des civilisations nègres dès son premier ouvrage en 1954, on peut suivre la relation de Senghor à l'histoire dans ses écrits, mais également durant les deux décennies où il exerça le pouvoir. Ce sont alors les politiques de la mémoire, depuis l'indépendance en 1960 jusqu'à aujourd'hui, notamment celles qui s'incarnent dans des lieux, des objets ou des pratiques du pouvoir - commémorative, législative — qui sont l'objet de l'analyse. C'est à partir de l'articulation de ces deux entrées sur le passé africain, vu depuis le Sénégal, qu'on saisit mieux l'imaginaire contemporain de la «Renaissance». En partant d'une contre-bibliothèque coloniale écrite dès la fin des années 1940, il s'agit moins de placer les enjeux sur des dynamiques politiques internes au Sénégal que sur l'actualité d'une définition de l'Afrique.

L'historicité du terme - la «Renaissance africaine » - formulée par Cheikh Anta Diop et Léopold Sédar Senghor, permet alors d'aborder une archéologie des régimes d'historicités panafricains. Le monument, la mémoire qu'il est censé véhiculer, s'appréhendant alors dans une réflexion plus globale dans les termes d'une philosophie de l'histoire.

\section{Deux définitions épistémologiques et politiques de l'Afrique dans les années qui suivent la Seconde Guerre mondiale}

Étudier la « Renaissance » dans une perspective historique, depuis le Sénégal, c'est s'intéresser au champ intellectuel sénégalais, à ce que l'on a pu désigner comme cette "démocratie des lettrés » (Diaw 1992). Point d'entrée de la colonisation française avec le système des comptoirs dès le XVII siècle, le pays a connu une trajectoire originale en Afrique occidentale. À la fin du $\mathrm{XIX}^{\mathrm{e}}$ siècle, le système des quatre communes instaure progressivement une citoyenneté pour certains résidents. Un peu plus tard, en 1914, l'élection de Blaise Diagne au parlement français est un événement majeur dans le monde de la politique française ${ }^{11}$. Premier député noir, il est percepteur de Senghor, quand jeune étudiant celui-ci débarque à Paris en 1928. Dans le

11. Pour une histoire détaillée de ces processus, voir JOHNSON (1991 [1971]). 
Paris des années 1920 émerge une communauté qui bientôt transforme, à une échelle mondiale, les représentations sur l'Afrique. Ce sont des artistes, des intellectuels noirs américains, des étudiants de l'Empire français qui donnent naissance, dans ce contexte d'effervescence culturelle, à ce que l'on nomme « la négritude ». Si l'invention du terme, formé du substantif « nègre », revient à Aimé Césaire (Senghor 1980), c'est Senghor qui en popularise l'usage. Pour Senghor (1964a: 9), la négritude représente «l'ensemble des valeurs du monde noir », selon une définition communément admise. Ce concept de la négritude est directement issu du mouvement de la Harlem Renaissance ${ }^{12}$. Dans cette définition culturelle de plusieurs identités sociales, le Martiniquais Césaire, le Guyanais Damas et le Sénégalais Senghor formulent une vision qui embrasse ainsi l'Afrique et sa diaspora, une conception intimement liée à une histoire appréhendée collectivement et dont le point de départ est la déportation esclavagiste de millions d'africains durant près de quatre siècles pendant la traite atlantique.

En 1945, une décennie après la parution du premier numéro de L'Étudiant noir, le monde est en ruine tandis qu'en Afrique noire francophone émerge une génération de militants politiques et d'intellectuels qui théorisent cette nouvelle situation historique. En fait, il faudrait plutôt parler de deux générations, celle du Rassemblement démocratique africain (RDA) - le grand parti fondé en 1946 - et celle d'une jeune génération d'étudiants qui arrivent en métropole. En 1945, Senghor est un homme qui a participé à la guerre. Repéré par Lamine Gueye alors maire de Dakar à près de 40 ans, il entre en politique, d'abord au sein de la Commission Monnerville chargée d'étudier la situation des territoires d'outre-mer lors de la constituante. Il le fait non au RDA — il n'assiste pas à son congrès, ce qu'il regrettera par ailleurs plus tard - mais dans ce qui est le grand parti hégémonique au Sénégal, la Section française de l'Internationale ouvrière (SFIO), dans sa branche sénégalaise. Senghor est élu député en octobre 1946 à la première Assemblée constituante, un poste de député au Palais Bourbon qu'il conserve jusqu'en 1960. L'année 1948 marque une rupture dans son itinéraire (Riesz 2011). Il publie à cette date l'Anthologie de la poésie nègre et malgache - livre aussi devenu célèbre, notamment pour un public français, pour la préface qu'en fit Sartre, Orphée noire. 1948 est aussi l'année où il crée son parti, le Bloc démocratique sénégalais (BDS), lequel change de nom plusieurs fois, en faisant alliance avec d'autres, pour enfin devenir le Parti socialiste dans les années 1960. Le Parti

12. Ce mouvement a pris forme dans le quartier de Harlem à New York. Impulsé par des artistes - écrivains, musiciens, peintres, etc. — et des intellectuels, il s'agissait d'affirmer des droits pour la communauté noire américaine. Voir KING (2015), l'un des ouvrages les plus récents sur ce mouvement. 
socialiste sera dominant sur la scène politique jusqu'à l'arrivée au pouvoir d'Abdoulaye Wade en 2000.

En 1948, un jeune Sénégalais de vingt-cinq ans est à Paris depuis deux ans : Cheikh Anta Diop. Il publie un article dans la revue le Musée vivant : «Quand pourra-t-on parler d'une renaissance africaine? » (Diop 1990 : 33-44). Dans ce contexte, la Renaissance européenne fait référence, bien sûr, à cette période de l'espace européen, qui l'a vu sortir du Moyen Âge. Par rapport aux thèmes développés par les étudiants noirs dans le Paris des années 1930, et inspirés de la Harlem Renaissance, un troisième terme a été rajouté, celui d'Afrique. Cela nous permet d'aborder ce moment historique d'une contre-invention de l'Afrique (Mudimbe 1994), c'est-à-dire une redéfinition de la «bibliothèque coloniale ». C'est bien par « la culture » qu'il faut donc lire la formulation d'un projet politique autant que d'une mémoire historique, l'énonciation du premier alimentant la seconde. Mais, au-delà de la culture, la question linguistique, ce premier procédé socio-symbolique humain, forme un cadre social originel de la mémoire collective (Halbwachs 1994). Chez les deux intellectuels sénégalais, cela renvoie à deux visions du monde.

\section{La langue du politique}

Dans ce court article de 1948, republié près de quarante ans plus tard par la maison d'édition ${ }^{13}$, dans le recueil Alertes sous les tropiques ${ }^{14}$, Cheikh Anta Diop n'explique pas précisément le terme de «Renaissance » mais ébauche plutôt un programme politique. Dès les premières lignes de son article, celui qui vient d'achever sa licence de philosophie s'en prend sans le nommer à son aîné, Senghor. Diop dénonce « celui qui écrit [et qui] cherche avant tout à s'exprimer correctement en français, à faire preuve d'un talent littéraire inattendu, de subtilités grammaticales, plutôt qu'à exprimer des idées utiles à sa collectivité » (ibid. : 34). Le centre de gravité de Cheikh Anta Diop, on le sait, ce sont les pyramides égyptiennes, et non pas un dialogue entre l'Occident et l'Afrique comme chez son compatriote; son œuvre est probablement la plus originale dans le domaine de la linguistique comparée, ou parenté génétique des langues (M'Bokolo 1996 : 12). Pour affirmer que les civilisations pharaoniques sont «nègres », Diop mène une démonstration sur les langues qu'il nomme ouloves ou valaf — c'est à dire wolof, la langue la plus parlée au

13. «Quand pourra-t-on parler d'une renaissance africaine?» paru d'abord dans Le musée vivant, numéro spécial, 36-37 (novembre 1948) : 97-165, republié dans Diop (1990 : 33-44). Sur l'histoire de Présence Africaine, on se reportera à FrIOUX-SALGAS (2009).

14. Le titre du recueil évoque bien sûr l'ouvrage de C. Lévi-Strauss. 
Sénégal. Mais à cet enjeu scientifique, il ajoute un questionnement politique. Les langues nationales sont « dans sa pensée politique un facteur stratégique de la libération des peuples africains et de leur unité culturelle [et il a ...] été constamment préoccupé par cette question », avance ainsi Boris Boubacar Diop (2006: 6). Ce même auteur ajoute: «La prise de conscience de cette dimension de l'être est indissociable de son idéal panafricaniste en ce sens qu'elle fonde l'unité culturelle du continent» (ibid. : 12). Diop revendique L'unité culturelle de l'Afrique noire, titre de son ouvrage paru en 1959, et cette démarche a des implications tout à la fois sur la définition du passé du continent comme sur son présent. Dans Les fondements économiques et culturels d'un État fédéral d'Afrique noire, paru en 1960, véritable manifeste, il énonce quinze points dans ses « conclusions pratiques $»^{15}$. Les deuxième et troisième points concernent cette question de la langue :

2) Travailler à l'unification linguistique à l'échelle territoriale et continentale, une seule langue africaine de culture et de gouvernement devant coiffer toutes les autres ; les langues européennes, quelles qu'elles soient, restant ou retombant au niveau de langues vivantes de l'enseignement secondaire ; 3) Élever officiellement nos langues nationales au rang de langues de gouvernement servant d'expression au Parlement et pour la rédaction des lois. La langue ne serait plus un obstacle à l'élection d'un député ou d'un mandataire analphabète de souche populaire (Diop 1974 [1960] : 120).

Se pose ici une première alternative au projet politique de Cheikh Anta Diop et de Léopold Sédar Senghor, le père de la francophonie. S'il est un domaine où l'on doit reconnaître la compétence de Léopold Sédar Senghor, c'est bien celui de la parole. Pour l'un de ses biographes, Senghor est ainsi « le maître de la langue » (Delas 2006). Senghor, qui obtient l'agrégation de grammaire en 1934, à sa troisième tentative, s'est préoccupé également très tôt de la question linguistique. Dans la première conférence qu'il donne à Dakar, en 1937, il évoque la question du «bicéphalisme», et l'un de ses thèmes majeurs est évoqué : " assimiler sans être assimilé ». Il interpelle le lecteur sur le bilinguisme : « Notre milieu n'est plus ouest-africain, il est aussi français, il est international ; pour tout dire, il est afro-français » (Senghor 1964a : 14). À ce constat, il ajoute :

Nous pouvons, à présent, poser ce principe général, que l'étude de l'Afrique occidentale et de la France doit constituer les deux pôles de l'enseignement en AOF et que ce

15. En note de bas de page est indiqué : « Ces 15 points constituaient l'essentiel des programmes du BMS et du FNS ». Le Bloc des masses sénégalaises (BMS) et le Front national sénégalais (FNS) furent deux partis créés au tout début des années 1960 par Cheikh Anta Diop. 
bicéphalisme ${ }^{16}$ se retrouvera à tous les degrés. À mesure que l'on avancera, le pôle Afrique perdra de sa force d'attraction au profit du pôle France [...]. C'est dire que bicéphalisme veut dire bilinguisme » (ibid.).

Plus de vingt plus tard, en 1958 à la veille des Indépendances, son article « Le problème des langues vernaculaires ou le bilinguisme comme solution » évoque cette conférence donnée à la Chambre de Commerce de Dakar en 1937. Il y réaffirme que « le choix de la langue d'enseignement est [...] le nœud du problème culturel pour tout pays sous-développé qui accède à l'autonomie » (ibid. : 228). Il développe ensuite l'un de ses thèmes majeurs, l'éducation :

Éduquer signifie, au sens étymologique du mot, «conduire hors de soi », hors de son milieu, transplanter. La vertu de l'éducation est de faire assimiler des richesses étrangères, dont on se fait un sang nouveau [...]. Si l'éducation est sortie de soi, elle est aussi enracinement (ibid. : 229).

L'enracinement est un des maîtres-mots de Senghor. Il a son pendant: l'ouverture. Dans cet article publié quelques mois avant que Charles de Gaulle propose son référendum à l'ensemble des territoires coloniaux, et où Sékou Touré déclare que les Guinéens préfèrent la liberté dans la pauvreté à la richesse dans l'esclavage, Senghor écrit : " En ce XX $\mathrm{XX}^{\mathrm{e}}$ siècle de l'interdépendance — nous n'avons pas attendu l'an de grâce 1958 pour prôner l'interdépendance et la Communauté France-Outre-Mer - le bilinguisme s'impose donc à nous » (ibid. : 230). Cette idée d'interdépendance constitue les prémisses de l'Eurafrique ${ }^{17}$. Dans une conférence qu'il donne en 1962, intitulée « Linguistique négro-africaine », il avance que « si l'Afrique de cette seconde moitié du Siècle doit s'enraciner pour se développer, c'est l'évidence qu'elle doit d'abord se connaître avant de s'accomplir : recenser ses richesses avant de les cultiver par l'assimilation de techniques étrangères » (ibid. : 330). Ainsi, chez Senghor, le « co-naître » (ibid. : 94) doit être appréhendé comme une conception de l'idée de « Renaissance ».

Avançons une première analogie sur cette conception épistémologique de la langue qui vaut, pour les deux auteurs, combat militant dans la définition d'un espace physique autant que mental : le continent africain. Ainsi, les pensées politiques de Senghor comme de Diop, liées à leur théorisation de cette question linguistique renvoient, pour reprendre les termes de Reinhart Koselleck (2011 [1997]), à des « horizons d'attente », soit l'articulation de

16. L'italique est la graphie utilisée par Senghor, comme dans les citations suivantes.

17. Ce thème est repris par Nicolas Sarkozy dans son « discours de Dakar» du 26 juillet 2007. Le texte se trouve en intégralité sur $:<\mathrm{http}: / /$ www.lemonde.fr/afrique/article/2007/11/09/ le-discours-de-dakar_976786_3212.html $>$. 
différents champs d'expériences collectives. Chez le premier, qui affectionne le fait de découper des mots, la juxtaposition de termes semble renvoyer à l'idée de différentes civilisations attenantes, ici l'européenne et l'africaine, qu'il s'agit de conjuguer dans une grammaire politique du métissage. Chez le second, l'attrait pour la génétique linguistique et la recherche d'une langue mère ${ }^{18}$ évoque bien sûr sa perception d'une unité historique et culturelle passée, qu'il s'agit de découvrir scientifiquement et de bâtir politiquement. Ici, l'« idée » de renaissance est densément — voire dangereusement, d'après les réactions de l'académisme français aux thèses de Cheikh Anta Diop ${ }^{19}$ stratégie et lutte politique.

\section{S'assimiler ou s'émanciper?}

En 1954, Cheikh Anta Diop était un étudiant brillant à Paris, il avait entamé une thèse avec deux sujets, comme cela se pratiquait à l'époque. La thèse principale, sous la direction de Gaston Bachelard, avait pour titre L'avenir culturel de la pensée africaine. La thèse secondaire, Qu'étaient les Égyptiens prédynastiques ?, était inscrite sous la direction de Marcel Griaule. Devant l'ampleur de ces vues, il ne parvint pas à réunir un jury et son travail fut publié dans ce qui reste considéré comme son ouvrage maître, Nations nègres et culture (Diop 1979 [1954]), le livre « le plus audacieux qu'un nègre ait jusqu'ici écrit» selon les mots d'Aimé Césaire (1955: 21). Diop y affirme ses thèses devenues célèbres depuis, concernant l'origine africaine, noire, des civilisations égyptiennes. Le débat tourne autour de la race et de la culture; des thèmes proches de ceux de Senghor. Mais à la différence de son article paru en 1948, Diop cite cette fois Senghor et l'une de ses phrases qui aujourd'hui encore reste parmi les plus controversées : «L'émotion est nègre, la raison est hellène. » Si Cheikh Anta Diop (1979: 55) souligne que ce vers est « d'une admirable beauté », c'est pour écrire juste après qu'il s'agit d'une « littérature nègre "de complémentarité" se voulant enfantine, puérile, bon enfant, passive, résignée, pleurnicharde » (ibid.). Le débat est lancé.

Il est vrai que Senghor a rendu les coups, mais sur le terrain politique plus que par la plume. En 1962, alors président, il envoie Cheikh Anta Diop pendant un mois en prison. Au lancement du Rassemblement démocratique

18. Ces travaux sont poursuivis par le linguiste, P. DiAGNe (2002).

19. L'épisode de sa soutenance de thèse en 1960 , où il obtient une mention seulement « passable », est connu ; le refus par la Sorbonne de lui décerner la plus haute mention représentait, de fait, une interdiction d'enseigner. Aujourd'hui encore, dans les couloirs des départements d'études africaines, en France, Cheikh Anta Diop provoque un haussement d'épaule doublé d'une moue dédaigneuse chez beaucoup de gens qui l'ont à peine lu. 
national (RND), en 1976, Siggi (se redresser en wolof), l'organe de presse du nouveau parti de Diop, est interdit par le grammairien et futur membre de l'Académie française, au motif d'une gémination abusive — le redoublement $\mathrm{du}$ « g » dans le titre du journal. Senghor avait utilisé ce même subterfuge contre le film Ceddo d'Ousmane Sembene. Toujours est-il que Cheikh Anta Diop, n'ayant cure de l'interdiction, lance immédiatement Taxaw (se tenir debout) ${ }^{20}$. C'est aussi qu'en 1976, une modification constitutionnelle signe le retour au multipartisme. Le président Senghor, seul chef d'État depuis $1962^{21}$ dans un régime semi-autocratique, décide d'autoriser trois courants politiques : le « communisme ou marxisme-léninisme », le « socialisme » et le «libéralisme» (Tine 1997). Le parti créé à cette époque par Cheikh Anta Diop, le RND, reste interdit ${ }^{22}$, tandis que cet événement consacre l'adoubement de Wade par Senghor (Diagne 2013). Mais si par certains côtés le régime de Senghor fut brutal, on ne peut lui reprocher d'avoir entretenu un culte du passé une fois au pouvoir. Ses usages de l'histoire semblent plus subtils, voire semblent relever du domaine de l'inconscient ou de l'impensé.

En 1948, est célébré le centenaire de l'abolition du 27 avril. Cette année-là, le jeune député du Sénégal signe une tribune dans le journal La Condition humaine qu'il vient de lancer : «La révolution de 1848 et le respect de la personne humaine $»^{23}$. Cet article reprend l'argument de la fin de l'esclavage comme acte du législateur français, en rappelant « la lucidité et [le] courage de quelques hommes à la tête desquels était Victor Schœlcher $»^{24}$, mais Senghor évoque un autre fait marquant de l'année 1848 : la publication du Manifeste du Parti communiste de Karl Marx et Friedrich Engels. Au lendemain de la Seconde Guerre mondiale, au Sénégal, le rappel de l'abolition de 1848 sert pour Senghor sa lutte contre un ordre social et politique, le colonialisme: « Nous savons, nous socialistes, et le "Manifeste" nous l'apprend que l'égalité politique — car les esclaves libérés devinrent citoyens français — n'est

20. On retrouve d'ailleurs ces polémiques et débats linguistiques, de haute tenue, dans les pages d'Àndë Sopi, le journal que lance en 1978 Mamadou Dia. Si Dia est membre fondateur du RND, il ne semble pas qu'il ait joué un rôle important dans ce parti ; libéré en mars 1974, il fonde la Coordination de l'opposition sénégalaise unie (COSU) qui visait à réunir une large coalition incluant les divers partis d'obédience marxistes (PAI, And-Jëf et LD/MPT).

21. La date de 1962 correspond à la crise de décembre 1962 où Senghor fit emprisonner le compagnon avec qui il avait cheminé depuis plus de quinze ans, le président du conseil, Mamadou Dia. Pour une analyse détaillée de cet événement, voir R. CoLIN (2007).

22. En effet, selon Senghor, le RND ne «rentrait» dans aucun des trois courants. Le RND ne sera autorisé qu'en 1981, alors que le nouveau président, Abdou Diouf, rétablit le multipartisme intégral.

23. Voir SENGHOR (1948).

24. Ibid. 
qu'illusion si elle n'est complétée par l'égalité sociale et l'instruction $»^{25}$. Senghor a une lecture pour le moins singulière de l'œuvre de Marx. Plusieurs articles, essentiellement dans Liberté II, Nations et voie africaine du socialisme, reviennent sur cette interprétation, celle du « jeune Marx ». Attardonsnous sur celui qui clôt le recueil, d'ailleurs intitulé «La voie africaine du socialisme ». Ce socialisme africain constitue le troisième terme, avec la négritude et la francophonie, de ce que Momar-Coumba Diop et Mamadou Diouf (1990 : 251) nomment le « triangle idéologique senghorien ». Dans cet article, Senghor $(1971: 285)$ revient d'abord sur son thème de prédilection, la culture : «l'indépendance culturelle est le préalable nécessaire aux autres indépendances : politique, économique et sociale »; ce qu'il rejette avant tout dans le socialisme scientifique, c'est son «matérialisme athée » (ibid. : 298). Senghor, ici, se désintéresse des questions économiques. Pourtant, dans le marxisme, l'économie (l'infrastructure) détermine ce qui est de l'ordre des représentations (la superstructure). En assignant à la culture un tel rôle, « en deçà et au-delà, au-dessus et au dedans de toutes les activités humaines : elle est l'esprit qui les anime, celui qui donne son style original à une civilisation » (ibid. : 295), Senghor inverse les termes principaux de la problématique du penseur de Trèves. Et cela a des implications sur le plan politique. Dans la suite de cet article, Senghor prône l'humanisme, mais un humanisme qui « exclut non seulement le malianisme, c'est l'évidence, puisque nous ne sommes pas que des Maliens ici rassemblés ${ }^{26}$, mais encore le nationalisme, le panégrisme — je ne dis pas la Négritude —, le panafricanisme, à plus forte raison le panarabisme » (ibid.). Si dans les années 1930, Senghor « traitait les Africains ralliés au marxisme de déserteur de la négritude » (Vaillant $2006: 320$ ), à la fin des années 1950 il cherche à élaborer une doctrine originale, celle d'un socialisme adapté aux réalités africaines. Mais, au début des années 1960, à l'heure des Indépendances, une autre question se pose : l'unité politique du continent. Concernant les États-Unis d'Afrique, il écrit : «Plusieurs raisons s'y opposent. La première est que le continentalisme est une forme d'autarcie [...]. On a parlé, par exemple, à la dernière conférence panafricaine de Tunis, de la création d'un Marché commun africain. A-t-on assez mesuré les difficultés de l'entreprise ? [...]. La seconde raison de la difficulté de l'entreprise est notre irréalisme, notre verbalisme. On vote des résolutions, qui ne sont pas suivies d'effets » (Senghor 1971 : 305). C'est vraisemblablement sur

25. Ibid.

26. Cet article est écrit au moment de l'éphémère Fédération du Mali, qui regroupait le Soudan français et le Sénégal et dura quelques mois en 1960. 
cette question que le désaccord avec Diop, et d'autres ${ }^{27}$, fut le plus lourd de conséquences. À lire parallèlement les écrits des deux penseurs sénégalais, on est frappé par ce «quelque chose de confus et de creux » (Diop 2006:4) chez le poète sérère, du moins ses écrits théoriques et politiques, opposé à la clarté de Diop. Citons les points cinq à sept du programme de Cheikh Anta Diop évoqués plus haut et qui aident à saisir le lien entre un projet politique et la tentative de réhabilitation d'une mémoire; Diop emploie le terme de conscience historique :

5) vivre l'unité fédérale africaine. L'unification immédiate de l'Afrique francophone et anglophone, seule pouvant servir de test. C'est l'unique moyen de faire basculer l'Afrique Noire sur la pente de son destin historique, une fois pour toutes. Attendre en alléguant des motifs secondaires, c'est laisser aux États le temps de s'ossifier pour devenir inaptes à la Fédération, comme en Amérique latine ; 6) Opposer une fin de non recevoir à toute idée de création d'États blancs, d'où qu'elle vienne et où que ce soit en Afrique Noire ; 7) Prendre dans la Constitution les dispositions nécessaires pour qu'il ne puisse pas exister une bourgeoisie industrielle. Prouver ainsi qu'on est réellement socialiste en prévenant l'un des maux fondamentaux du capitalisme. Qui pourrait aujourd'hui s'opposer décemment à une mesure préventive contre une classe inexistante en Afrique ? (Diop 1974 : 121).

C'est à la lecture de ces formulations que l'on saisit mieux ce que Cheikh Anta Diop attache au terme de «Renaissance africaine». Senghor, comme Diop, ont lu les écrits de Marx et de Lénine et ils ont été inspirés par ces penseurs. Le titre du dernier ouvrage de Cheikh Anta Diop publié en 1981 reprend même une alternative formulée par Léon Trotsky pendant la Première Guerre mondiale : civilisation ou barbarie. Mais si les deux reconnaissent l'apport du matérialisme dialectique, chacun fait un écart avec la théorie marxiste, Senghor en y ajoutant de l'humanisme chrétien, Diop en puisant dans des sources panafricaines.

En un sens, certains de ces débats s'étaient exprimés en 1956, lors du premier Congrès des intellectuels noirs à la Sorbonne. Ce congrès marque à la fois un tournant décisif dans l'affirmation d'une identité noire, mais aussi une vraie rupture idéologique sur plusieurs plans, et notamment politique. Aux questionnements sur la culture succèdent ceux sur les rapports sociaux de production : l'identité noire doit être pensée à l'intérieur du monde colonial et impérialiste. Ce congrès se tient un an après la Conférence de Bandoeng, probablement l'événement historique le plus important $\mathrm{du} \mathrm{xx}^{\mathrm{e}}$ siècle pour les peuples non occidentaux. Senghor, d'ailleurs, n'hésite pas à dire que depuis la Renaissance « aucun événement n'a revêtu l'importance historique du coup

27. Ces deux visions allaient s'exprimer dans les groupes d'États dits de Casablanca et ceux de Monrovia, voir BouKARI-YABARA (2014 : 186-188). 
de tonnerre de Bandoeng 》 (Dieng 2007 : 119). Ainsi, ce temps des options politiques où s'esquisse un paradigme original dans un monde bipolaire, est celui de la formulation de nouveaux référents idéologiques, conceptuels, et bientôt normatifs. Aux débats d'idées s'imbriquent progressivement des usages canoniques du passé. Alioune Diop, le Sénégalais, fondateur de Présence Africaine, qui a initié cette rencontre, a invité deux de ses jeunes compatriotes étudiants à présenter une communication: Cheikh Anta Diop et Abdoulaye Wade. Saisir l'imaginaire du Monument de la Renaissance à partir du milieu des années 2000 impose de s'intéresser, plus largement, au rapport aux passés de l'étudiant Wade, dans les années 1950, puis de l'opposant politique Wade, à partir des années 1970 et surtout 1980. On doit alors tenter de démêler les fils entre ce qui renvoie à des références explicites, ce qui semble relever d'une mémoire historique déjà instituée et ce qui apparaît comme apport original.

La question du passé collectif, de l'histoire et de la mémoire, hier et aujourd'hui au Sénégal

Cheikh Anta Diop et Léopold Sédar Senghor ont produit deux œuvres considérables. Ils se sont posé des questions à propos de la colonisation, comme on vient de le voir, mais aussi à propos d'une période plus ancienne : la traite transatlantique. On retrouve leurs réflexions, plus ou moins explicitement, dans la mise en pratique d'une gouvernance de la mémoire par Abdoulaye Wade, un domaine où il a eu une activité conséquente en tant que président à partir de 2000, et qui aboutira en 2010 à l'inauguration du Monument de Renaissance. Mais pour mieux saisir la production du passé par Wade en ce début de XXI siècle, et ainsi comprendre « son » idée de la «Renaissance», il nous faut au préalable faire un détour par son itinéraire intellectuel et politique.

Abdoulaye Wade est à Paris au début des années 1950. En 1953, Présence Africaine publie l'un de ses articles — où il fait référence, dès les premières lignes, à Cheikh Anta Diop - intitulé « Afrique noire et Union française ». Dans cet article d'une trentaine de pages, dont il dira cinquante ans plus tard qu'il n'est « pas très fier [de l'] évoquer aujourd'hui essentiellement d'ailleurs pour des questions de style car sur le fond [ses] options ont très peu changé » (Wade 2008 : 65), Wade passe en revue le passé « précolonial » de l'Afrique de l'Ouest, sa «découverte», la « colonisation», les «politiques d'association », l'«Union française », enfin les «perspectives d'avenir ». À vrai dire, à lire ce tableau général, on ne saisit pas nécessairement où l'auteur veut en venir. D'ailleurs, ce n'est pas faire insulte à Abdoulaye Wade que de dire que sa pensée théorique n'a jusqu'à présent pas retenu la même attention 
que celles de Diop et de Senghor. Wade exprime d'abord ses vues dans sa thèse d'économie publiée en 1964 : Économie de l'Ouest-africain. Unité et croissance. La problématique centrale qu'il développe porte sur l'importance de prendre en compte, dans l'analyse macro-économique, ce qu'il nomme des «effets de percussion». Ce nouveau vocabulaire qu'il s'ingénie à formaliser provient de la mécanique; il devrait, dans les idées avancées par l'auteur, permettre de mieux situer des causes de la croissance économique. Si ces notions n'ont pas connu de succès dans les sciences économiques, on doit en revanche souligner l'un des paradoxes de l'ouvrage. Wade aussi a lu Marx. En prenant l'exemple de la Russie d'après la révolution d'Octobre, il reconnaît que le système marxiste a fourni " les meilleurs résultats en matière de développement économique de pays attardés » (Wade $1964: 319$ ). Pourtant, dans le cas des économies ouest-africaines, il souhaite y adjoindre, en favorisant l'investissement de capitaux étrangers, une touche de libéralisme. Wade, dans ces années-là, milite au sein de l'Association des étudiants du rassemblement démocratique africain (AERDA) dont le Secrétaire général est Cheikh Anta Diop. Il flirte ensuite avec la Fédération des étudiants d'Afrique noire française (FEANF), la grande centrale étudiante fondée en 1950 et qui aura un rôle décisif dans la formulation de revendications indépendantistes. Il sera l'un des responsables de section, sans être toutefois un militant de premier plan. Diop écrit :

[...] les congrès de la FEANF organisés à Paris nous donnaient toujours l'occasion de grands débats sur toutes les questions, pendant des journées et des nuits entières, qui à chaque fois voyaient s'opposer marxistes et non marxistes. Mais j'arrivais à avoir les marxistes à l'usure. Je me souviens notamment d'une discussion sur une résolution qui dura jusqu'à quatre heures du matin, sans solution. Tout le monde était épuisé. Je me retirais alors et m'enfermais dans les toilettes, où je dormis quelques minutes. Je regagnais ensuite la salle, tout frais, ce qui me permit de dominer tous ceux qui s'élevaient contre mon texte et de parvenir à le faire voter (Wade $2008: 66$ ).

$\mathrm{Du}$ débat en question on ne saura rien, mais on voit là le «style » d'Abdoulaye Wade, notamment l'omniprésence du « Je ». S'il s'agit d'une anecdote, elle semble révéler la capacité du troisième président du Sénégal à se raconter, à édifier sa propre stature politique, plutôt que l'étendue de ses idéaux politiques.

Abdoulaye Wade a gouverné le Sénégal pendant douze années. Comment expliquer cette longévité politique ? Comme le note Pathé Diagne (2013 : 102), il « devra sa réussite au hasard des événements, mais aussi sur le plan personnel, à cette habileté et cette souplesse décelées par Senghor qui l'identifiait 
à Njomboor, l'assimilant ainsi au personnage de Leuk-le-Lièvre aux milles tours et facettes, qui personnifie la Ruse des contes wolof de Sadji et Senghor ou ceux de Birago Diop ». Dans cet article intitulé "Abdoulaye Wade ou la fin du cycle senghorien », Diagne explique comment Senghor a construit cet opposant, sûrement celui qu'il pouvait le mieux « contrôler » face à des hommes de la trempe de Cheikh Anta Diop, mais aussi d'Abdoulaye Ly ou de Mamadou Dia, pour ne citer que ces deux illustres noms. Wade fera fructifier ce legs sous Abdou Diouf, avant de réussir à être élu président en 2000. Divers travaux ont mis en lumière la personnalité, l'itinéraire ou la politique d'Abdoulaye Wade, sous différents aspects (Diop 2013a, b) ${ }^{28}$. Ce qui nous intéresse ici, c'est plutôt comment il s'est employé à élaborer symboliquement du passé, à construire de la mémoire. Il nous faut alors comprendre comment ces discours publics sur l'histoire au Sénégal ont, historiquement, aggloméré différentes références. Ces références sont d'abord coloniales et ouest-africaines, mais on peut aussi les appréhender à partir d'un espace globalisé de la mémoire, en se centrant alors sur un passé africain « immémorial» : la traite esclavagiste transatlantique.

\section{Résister}

Lat Dior Diop, roi du Sénégal avec le titre de Damel, en 1883, écrivit aux Français : « Tant que je vivrai, je m'opposerai de toutes mes forces à l'établissement de ce chemin de fer..., je te répondrai toujours non, non, non. Quand bien même je dormirais, mon cheval Malaw te ferait la même réponse ». Après une célèbre résistance Lat Dior fut vaincu et tué à Dekhelé (Wade 1989 : 91).

Ces lignes sont tirées de l'ouvrage d'Abdoulaye Wade, Destin pour l'Afrique, lequel défend la résistance des peuples africains. Le chapitre-pivot du livre s'intitule d'ailleurs « Permanence de la résistance. Composante essentielle du panafricanisme ». Dans cet ouvrage, Wade cherche à construire son rôle d'opposant au pouvoir en place. On peut alors s'interroger sur les comparaisons qu'il propose entre sa propre carrière politique et la figure de Lat-Dior au plan national. Cette figure avait été largement édifiée lors des commémorations du centenaire de la mort du héros en 1986 et c'est à cet imaginaire historique que l'on doit maintenant s'intéresser.

Lat-Dior, personnage historique né en 1842, mort en 1886, devint après les Indépendances la figure principale de ce que l'on présente comme la

28. Ces deux ouvrages collectifs sont des sommes considérables — plus de deux fois huit cents pages - indispensables pour comprendre le Sénégal de la première décennie 2000. 
résistance à la colonisation. Ce processus intervient déjà sous Senghor. Si on a pu noter que " producteur d'idéologie, L. S. Senghor dénia à l'histoire un rôle quelconque dans la recréation culturelle de l'Afrique » (Diop \& Diouf 1990 : 271), on doit faire une remarque complémentaire : c'est par la culture que le régime de Senghor a recréé de l'histoire (Mourre 2017a). En octobre 1986, nous assistons donc aux commémorations du centenaire de la mort de LatDior, qui furent un véritable événement au Sénégal. La presse offre un bon aperçu de la consistance de ce passé. La Une de l'édition spéciale du Soleil, le grand quotidien national, indique « Dekeulé 100 ans après » à l'encre rouge dégoulinante, évoquant le sang du héros. Les titres des articles renvoient à l'hyperbole attachée à cette figure : «La référence $»^{29}$, «Le sens d'un sacrifice ${ }^{30}$ », « Mystique du héros ou la quête de la référence ${ }^{31}$, «Un damel sur le chemin de l'honneur $»^{32}$, «Résistance des armes, résistance des âmes $»^{33}$, «Lat-Dior, sans peur et sans reproche ${ }^{34}$, etc. Dans l'éditorial introduisant ce numéro spécial, on lit:

Lat-Dior héros national ! C'est assurément un choix judicieux, car le Damel incarna, au plus haut point, ces vertus qui sont la sève nourricière de notre fierté incommensurable d'être les descendants de nos ancêtres, d'être des Sénégalais ${ }^{35}$.

En 1986, on évoque ce chef qui « incarne l'unité nationale $»^{36}$ et, dans le cadre de ces commémorations officielles, Abdou Diouf veut « faire de chaque Sénégalais un Lat-Dior » ${ }^{37}$. Il entend par là « avant tout regarder vers l'avenir et affronter avec nos propres armes, les défis du présent : le sous-développement et les tristes imprécations, le système inique de l'apartheid et les manœuvres de ceux qui veulent détruire des civilisations bâties avec patience, amour et peine $»^{38}$. Ainsi, la commémoration apparaît comme un lieu idéal pour cerner comment ce passé est « inventé » et, en ce sens, pour discutables qu'ils soient

29. A. Dramé, «La référence », Le Soleil, 25-26 octobre 1986, p. 10.

30. E. B. Sow, « Le sens d'un sacrifice», Le Soleil, 25-26 octobre 1986, p. 10.

31. B. Diouf, « Mystique du héros ou la quête de la référence », Le Soleil, 25-26 octobre 1986, pp. 10-11.

32. I. Fall \& E. B. Sow, « Mamadou Diouf. Un damel sur le chemin de l'honneur », Le Soleil, 25-26 octobre 1986, p. 10.

33. A. N. Sylla, « Résistance des armes, résistance des âmes », Le Soleil, 25-26 octobre 1986, p. 15.

34. A. Fall, «Lat-Dior, sans peur et sans reproche », Le Soleil, 25-26 octobre 1986, p. 15.

35. A. Dramé, «La référence », Le Soleil, 25-26 octobre 1986, p. 10.

36. I. D. Thiam, «Lat-Dior incarne l'unité nationale», Le Soleil, 25-26 octobre 1986, p. 22.

37. Non signé, «Faire de chaque sénégalais un Lat-Dior pour vaincre le sous-développement», Le Soleil, 28 octobre 1986, p. 2.

38. Ibid. 
sur un plan historique (Diouf 1990), les propos de l'opposant A. Wade dans son ouvrage de 1989 ne font que reprendre un langage commun du nationalisme sénégalais largement diffusé au milieu des années 1980. Notons par ailleurs qu'à cette époque une statue, non pas de Lat-Dior mais de son cheval Malaw, avait été érigée dans le centre-ville de la capitale sénégalaise. Vingt ans plus tard, une fois à la tête de l'État, le président Wade s'est réapproprié ce langage. Mais de manière significative à travers une autre « figure », celle qui devient un personnage historique au singulier : «le» tirailleur sénégalais. On peut ici lire ces mots de la «Résistance» — qui bientôt seront ceux de la «Renaissance» à travers l'ensemble statutaire Demba et Dupont.

Wade s'est ainsi attaché à créer d'autres héros, ceux qui d'ailleurs, historiquement, avaient participé à vaincre sur les champs de bataille le Damel du Cayor, les tirailleurs sénégalais. On se concentrera ici sur la première célébration de la Journée du tirailleur, le 23 août 2004 ; l'année 2004 marquant le soixantième anniversaire du débarquement de Provence, où les troupes coloniales jouèrent, signalons-le, un rôle décisif dans la libération du territoire français. Ce jour-là, l'événement fait la Une des quotidiens nationaux ${ }^{39}$. Le quotidien Le Populaire titre ainsi : «Enfin l'hommage aux tirailleurs » ${ }^{40}$, tandis que Wal Fadjri avance: "Ce que Senghor et Abdou Diouf auraient dû faire $»^{41}$. Le journal Le Soleil titre le 25 août 2004 : «Bravo Wade » ${ }^{42}$. L'un des moments forts de cette journée fut la réhabilitation de l'ensemble statuaire Demba et Dupont, cette stèle symbolisant un soldat africain et un soldat français qui avait été inaugurée en 1923 au lendemain de la Première Guerre mondiale, puis déboulonnée soixante ans plus tard, une nuit d'août 1983, sous Abdou Diouf, justement parce qu'elle était considérée comme un symbole du paternalisme colonial français ${ }^{43}$. La statue avait alors été remisée dans les allées d'un cimetière de la capitale sénégalaise. Comme le souligne Ferdinand De Jong (2008), la (ré)apparition de cet ensemble statuaire a modifié le paysage mémoriel, colonial, de la cité dakaroise. Cet usage du passé n'en est pas moins ambivalent. Ce 24 août, on insiste sur le destin commun «franco-africain» que symbolisent ces deux noms ${ }^{44}$. Pour Wade : « la volonté de l'artiste [était] d'inscrire dans le volume et la forme, pour les

39. «La Journée du tirailleur à la une des quotidiens du lundi », APS, 23 août 2004, <http:// archives.aps.sn/article $/ 1263$ ? lightbox $\% 5$ Bwidth $\% 5 \mathrm{D}=75$ p\&lightbox $\% 5$ Bheight $\% 5 \mathrm{D}=$ $90 \mathrm{p}>$.

40. Ibid.

41. Ibid.

42. Non signé, « Bravo Wade», Le Soleil, 25 août 2004, p. 3.

43. Le journal Le Soleil fait d'ailleurs sa Une sur cet « enlèvement». Voir «Plus de statue place Tascher », Le Soleil, 17 août 1983, p. 1.

44. Pour une analyse spécifique de cette journée, voir F. DE Jong (2008). 
générations futures, les combats communs de Français et d'Africains qui ont contribué à instaurer ce monde d'aujourd'hui dont nous sommes fiers $»^{45}$. Les deux autres chefs d'État présents ce jour-là devant cet ensemble statutaire, le Malien Amadou Toumani Touré et le Burkinabé Blaise Compaoré, ont également salué l'initiative de Jacques Chirac de commémorer le débarquement de Provence sur le porte-avions Charles de Gaulle dans la rade de Toulon quelques jours plus tôt. Le monument, ici, s'inscrit dans des enjeux géopolitiques contemporains tout en renvoyant, de facto, à une histoire franco-africaine plus longue ; une histoire qui prend notamment corps après la Première Guerre mondiale et qui s'est matérialisée dans plusieurs statues (Mann 2005) ${ }^{46}$, une histoire qu'Abdoulaye Wade connaît intimement, son père ayant été un vétéran de la Première Guerre mondiale. En 2006, la poste lançait, "sur recommandation des autorités sénégalaises ${ }^{47}$, un timbre à l'effigie de Demba et Dupont. La mémoire historique, et l'imaginaire qu'elle charrie, semble osciller, ici, dans ses supports mêmes, entre grandeur et fixité du monument sur la place publique, d'un côté, volatilité et intrusion dans les foyers, de l'autre. Ces commémorations étatiques on était saluées dans la presse, comme le montrent certains titres plein d'emphase: «Pour la gloire et pour l'immortalité $»^{48}$; «Une si longue et fabuleuse histoire $»^{49}$; «Pour une réécriture de l'histoire $»^{50} ;$ «La réécriture de l'histoire commence... $»^{51}$; «Le rôle des Tirailleurs revisité » ${ }^{52}$; «Un devoir de mémoire et de vérité historique $»^{53}$. Ce répertoire constitue une forme de récit dominant. Si les thèmes principaux sont ceux de « la chair à canon » durant la Première Guerre mondiale, des exactions de l'armée allemande lors de la Seconde, et enfin de la cristallisation des pensions financières des anciens combattants lors des Indépendances, cette production du passé rappelle parfois celle qui était intervenue vingt ans plus tôt avec la figure de Lat-Dior. Ici, dans cette longue

45. M-L., Badji, « Dupont et Demba, ciment d'un avenir commun », Le Soleil, 24 août 2004, p. 4.

46. À la même époque, une réplique de la statue Demba et Dupont fut construite à Reims — puis détruite par l'armée allemande en 1940 — tandis qu'une autre statue commémorant le sacrifice des troupes noires était inaugurée à Bamako.

47. Non signé, «Lancement d'un timbre-poste à l'effigie de Demba et Dupont », Agence de presse sénégalaise, 21 août 2006, < http://archives.aps.sn/article/21797?lightbox\%5Bw idth $\% 5 \mathrm{D}=75 \mathrm{p} \&$ lightbox $\% 5$ Bheight $\% 5 \mathrm{D}=90 \mathrm{p}>$.

48. B. Diouf, « Pour la gloire et l'immortalité », Le Soleil, 24-25 mai 2008, p. 1.

49. F. Sambe, «Une si longue et fabuleuse histoire», Le Soleil, 23 août 2008, p. 13.

50. I. Ndiaye, «Pour une réécriture de l'histoire», Le Soleil, 24-25 mai 2008, p. 5.

51. M. L. Badji, «Dupont et Demba, ciment d'un avenir commun », Le Soleil, 24 août 2004, p. 4.

52. O. Ndiaye, «Le rôle des tirailleurs revisité », Le Soleil, 24 août 2004, p. 3.

53. B. Diallo, «Un devoir de mémoire et de vérité historique », Le Soleil, 24 août 2004. p. 5. 
durée de l'iniquité et de l'oppression, le débat semble s'articuler autour d'un paradigme principal : la réhabilitation des anciens combattants, et plus généralement de l'histoire africaine.

Cette écriture semble participer d'une tentative de renforcement de la cohésion nationale, moins que d'une restauration continentale comme chez Cheikh Anta Diop, même si certains thèmes s'en rapprochent. Le courage, l'honneur, la tradition, voire la noblesse, sont des valeurs qui reviennent régulièrement lorsqu'on évoque les tirailleurs. Dans les années 2000 « l'objet tirailleur », après Lat-Dior dans les années 1980, semble devenir un objet omnipotent, un objet de désir, un fétiche de la postcolonialité sénégalaise, pour reprendre les termes d'Achille Mbembe (2005). Mais comprendre la densité de la mémoire historique de ce présent sénégalais, et de ce qui relève de l'imaginaire collectif, implique de se pencher également sur un temps long, cette fois continental : celui qui concerne la traite négrière.

\section{Du débat des réparations à celui de la Renaissance}

Devant les vicissitudes de l'histoire et les difficultés économiques beaucoup d'Africains sont en proie au doute, et s'interrogent avec angoisse sur leur devenir. Et pourtant, dans «Un destin pour l'Afrique », Maître Abdoulaye Wade met en perspective l'élan de fraternité humaine se substituant à l'insoutenable commerce triangulaire, à travers trois personnages s'élançant l'un vers l'autre. Cette idée est la source d'inspiration du Monument de la Renaissance africaine (homme, femme, enfant) qui se libère des pesanteurs et entraves, pour s'approprier son destin, construire son avenir, à la conquête du mieux-être, symbole de l'africain.

Ces mots sont ceux du décret $\mathrm{n}^{\circ} 2003-593$ du 16 juillet 2003 portant création du Monument de la Renaissance africaine. Ce récit sur l'esclavage est concomitant de celui qui concerne « le» tirailleur, il a pourtant une dynamique propre. Pour le comprendre, on peut se pencher d'abord sur le cas de Gorée, un lieu d'observation privilégié pour saisir un rapport national à un passé transnational.

La «fabrication d'un patrimoine» à Gorée (Bocoum \& Toulier 2013) s'est réalisée dès la période coloniale, notamment après la Première Guerre mondiale. Mais c'est après l'indépendance d'avril 1960 que Gorée s'impose progressivement comme symbole de la Traite à une autre échelle, un symbole qui s'exprime par la culture et qui touche l'ensemble du «monde noir». En 1966, Senghor organise le Festival mondial des arts nègres (FESMAN) (Ficquet $\&$ Gallimardet 2009). Lors de la grande manifestation culturelle et intellectuelle 
de la négritude, où par ailleurs Cheikh Anta Diop est reconnu comme l'intellectuel africain le plus fécond du XX ${ }^{e}$ siècle, avec W. E. B. Dubois, l'île connait un nouvel essor. Pendant trois semaines, chaque soir, a lieu un spectacle « Son et Lumière ». L'image de Gorée se renforce comme celle du lieu de la mémoire de la traite atlantique sur le continent africain. En 1978, l'île de Gorée est inscrite sur la liste du patrimoine mondial de l'UNESCO, « officialisant » ce nom comme étant synonyme de la traite atlantique. Dans les rapports des experts de l'UNESCO, l'île devient cette image « du choc de deux civilisations différentes, et le témoin d'une expérience humaine sans précédent dans l'histoire des peuples $\|^{54}$. Dans ces lignes, la traite atlantique n'est pas nommée et n'est évoquée que par un biais qui est d'abord celui de l'émotion — en témoignent des termes comme «choc » ou " expérience ». Dans ce même document, on se place à un niveau qui est celui de « la conscience universelle » ${ }^{55}$, Gorée est évoquée comme lieu d'un «cortège de souffrance, de larmes et de mort $»^{56}$. De plus, «l'attention que les autorités sénégalaises portent à cette île ne se réfère pas à un désir de s'attarder sur un passé à jamais enfoui mais à une volonté de faire de l'île de Gorée l'archétype de la souffrance de l'homme noir à travers les âges $\rangle^{57}$. Expliquer la traite atlantique, dans ce cadre, c'est se référer à « l'aveuglement et la haine [qui] ont naguère sévi » ${ }^{58}$. S'intéresser à Gorée aujourd'hui contribue alors à réaliser une histoire des idées et des imaginaires qui se rattachent à l'esclavage et à une réflexion plus générale sur la mémoire actuelle de la traite atlantique participant d'une « dialectique de la diversité mémorielle » (Bassène 2011), dont il s'agit de définir la trajectoire, les contours comme la densité ${ }^{59}$. La teneur des rapports de l'UNESCO est surprenante (Mourre 2018). En 1998, on encourage le gouvernement à continuer le développement touristique et on y présente l'île comme la "principale destination touristique $»^{60}$ du pays. L'année suivante, le président sénégalais Abdou Diouf inaugure la réplique du Mémorial Gorée-Almadies, ce mémorial

54. Formulaire de proposition d'inscription, in « Dossier de nomination de l'île de Gorée », UNESCO CLT/WHC/Gorée/14.

55. Ibid

56. Ibid.

57. Ibid.

58. Ibid.

59. Notons que c'est au nom du critère vi (6) que l'île de Gorée, comme le camp d'AuschwitzBirkenau un an plus tard, est inscrite sur la liste du patrimoine mondial. Ce critère précise que le site doit «être directement ou matériellement associé à des événements ou des traditions vivantes, des idées, des croyances ou des œuvres artistiques et littéraires ayant une signification universelle exceptionnelle », voir < http://whc.unesco.org/fr/criteres/>.

60. Voir les «Documents examinés par le comité» relevant du «Point 7 » de l'ordre du jour lors de la $22^{\mathrm{e}}$ session du Comité du patrimoine mondial à Kyoto au Japon (du 30 novembre au 5 décembre, 1998) où l'état de conservation de chaque propriété de la 
qui devait se construire à Dakar, dans le quartier des Almadies, en face de l'île. La construction de ce mémorial avait été décidée à la fin des années 1980 mais sa réalisation a toujours été ajournée depuis, symbolisant peut-être les hésitations des politiques mémorielles de l'État sénégalais. Cette colonne représentant la voile d'un bateau négrier, située sur la partie la plus haute de l'île de Gorée, a d'ailleurs fait l'objet, à la fin des années 2000, de nombreuses critiques de la part de l'UNESCO, au motif qu'elle dénaturait le site.

Abdoulaye Wade arrive au pouvoir en avril 2000. Un an plus tard, se tient la conférence de Durban « contre le racisme, la discrimination raciale, la xénophobie et l'intolérance » au pays du président Thabo Mbeki qui vient de lancer ce slogan de la Renaissance africaine. Ici se scelle probablement le nouvel ancrage de Wade. À la résistance, il amarre la Renaissance. Cette conférence reconnaît dans sa déclaration finale, un an après le vote de la loi Taubira en France, l'esclavage comme un crime contre l'humanité. À Durban, les débats concernant notamment la question des réparations ont été vifs. Dans un contexte où Wade lançait avec d'autres pays africains — l'Afrique du Sud, l'Algérie, l'Égypte et le Nigeria - le Nouveau partenariat pour le développement de l'Afrique (New Partnership for Africa's Development, NEPAD), il considérait que des réparations financières n'avaient pas leur place. À Durban, il concluait ainsi son discours : « Le peuple africain est le peuple qui a été le plus agressé dans l'histoire, l'esclavage, la colonisation, l'exploitation et maintenant la dette. Avec tout cela il est encore debout. Voici pourquoi son génie qui l'a protégé jusqu'ici, le fera survivre ${ }^{61} »$. Si Wade s'est peu intéressé à entretenir l'île de Gorée, occupé par d'autres chantiers sur le continent, il faut noter un élément important durant son mandat quant à la mémoire de l'esclavage : le vote d'une loi mémorielle en 2010. En effet, au moment du cinquantenaire des Indépendances, le parlement sénégalais adopta une loi qui déclarait dans son article 1 «que l'esclavage et la traite négrière, sous toutes leurs formes, constituent un crime contre l'humanité $\rangle^{62}$, devenant donc le

Liste est considéré dont l'île de Gorée, «État des biens inscrits sur la liste du patrimoine mondial » (<WHC-98/Conf.203/08Rev>, p. 34 (document en anglais), pour la traduction française en ligne : $<$ http://whc.unesco.org/fr/soc/2272>, p. 64).

61. Ibid.

62. L'article 2 avance que : «La présente déclaration solennelle sera commémorée chaque année sur toute l'étendue du territoire national, le 27 avril, correspondant à la date de l'abolition de la traite négrière dans les colonies françaises, en 1848, à l'initiative de Victor Schœlcher ». L'article 3 énonce que : «Les programmes scolaires devront, notamment, dans les cours d'histoire, inclure cette question et lui réserver suffisamment de place pour que nos enfants comprennent bien ce qui s'est passé et les conséquences de la traite négrière sur l'évolution de l'Afrique. » Loi 2010-10 du 5 mai 2010 déclarant l'esclavage et la 
second pays au monde à adopter un tel texte. Dans les exposés du motif de la loi, qui rappelait le précédent de la loi Taubira, il était écrit que :

Sans s'attarder sur la question des réparations pécuniaires incompatibles avec notre dignité, il est important que chaque élève, dans chaque école du monde et que chaque peuple partout où il se trouve intègre cette problématique dans l'Histoire de son pays et dans ses relations avec les autres, apprenne l'histoire de la traite négrière et celle de l'esclavage, pour en tirer des leçons, aux fins de bâtir un monde respectueux de l'espèce humaine, sans considération de race, d'ethnie ou autre ; un monde de liberté, de justice, de reconnaissance mutuelle, fondé sur l'égale dignité des peuples et des cultures, la conscience claire que la civilisation de l'Universel est un faisceau d'apports successifs, dans lequel, chaque État, si petit soit-il, a sa part de contribution ${ }^{63}$.

Lors de l'examen de cette loi et dans son rapport, il était pourtant souligné que :

certains commissaires se disent perplexes et doutent de l'utilité et de l'opportunité de cette loi. Pour eux, il ne sert à rien de pleurer sur son sort et de ressasser le passé à l'heure de la Renaissance africaine. En outre, ils ont posé le problème de la responsabilité après toutes ces générations passées et posent la question de savoir : «si on ne réclame pas de réparations, qu'est-ce qu'on attend à travers cette loi ? ${ }^{64}$

Un sénateur, Pathé Guissé, émit le souhait d'une loi déclarant la colonisation comme un crime contre l'humanité, mais également que dans le futur, le présent — qui serait alors du passé — soit jugé :

La colonisation a déstructuré toutes nos sociétés, nos royaumes. Peut-être qu'un jour le Parlement votera une loi déclarant la colonisation crime contre l'humanité [...]. Nous espérons également que la jeunesse africaine libérée de certaines contraintes votera un jour une loi criminalisant le néo-colonialisme et les plans d'ajustement structurel ${ }^{65}$.

C'est ici la question du passé en tant que tel qui semble constituer un horizon d'attente, plus que des luttes présentes, et le Parlement, avec ses

traite négrière, crimes contre l'Humanité (Journal Officiel de la République sénégalaise, $\mathrm{n}^{\circ}$ 6560, 11 décembre 2010).

63. Exposé des motifs de la Loi 2010-10 du 5 mai 2010 déclarant l'esclavage et la traite négrière, crimes contre l'Humanité (Journal Officiel de la république sénégalaise, $\mathrm{n}^{\circ} 6560$, 11 décembre 2010).

64. Rapport de la loi déclarant l'esclavage et la traite négrière crimes contre l'Humanité.

65. P. Guissé, cité dans «Un sénateur préconise de criminaliser la colonisation », APS, 27 avril 2010. 
propres règles, est un des lieux de cette activité politique mémorielle renvoyant à des gouvernances — plus ou moins abouties — d'imaginaires collectifs.

En 2003, l'article premier du décret officialisant la création du Monument de la Renaissance stipulait qu'il est :

érigé sur la colline dite de la deuxième Mamelle sise à Dakar, un Monument de la Renaissance africaine qui traduit la volonté des peuples noirs, victimes des plus grandes tragédies humaines, de se réapproprier leur destin en triomphant de l'esclavage, de la colonisation et de la dictature. Il exprime l'Afrique qui surgit de l'obscurantisme, des fléaux et des préjugés ${ }^{66}$.

Cette conception de l'histoire du continent semble en partie réifier certaines dynamiques. Dans sa Critique de la raison nègre, Achille Mbembe (2013 : 142) avance que «l'Afrique n'existe qu'à partir d'une bibliothèque coloniale qui s'immisce et s'insinue partout, y compris dans le discours qui prétend la réfuter, au point où, en matière d'identité, de tradition ou d'authenticité, il est sinon impossible du moins difficile de distinguer l'original de sa copie, voire de son simulacre ». À l'heure actuelle, alors qu'Abdoulaye Wade a quitté le pouvoir depuis 2012 après sa défaite aux élections présidentielles, les polémiques sur le monument semblent s'être estompées, et personne au Sénégal ne songe réellement à le faire détruire ${ }^{67}$. Or, « l'idée » de la « Renaissance » est toujours présente dans les discours publics. Au cours de l'année 2016, sur le site de l'Agence de presse sénégalaise, plus de vingt-cinq articles reprenaient ce thème, en l'appliquant à la religion, à la science, au sport, à la culture, à l'éducation, etc. Pourtant le terme, rarement défini, semble aujourd'hui être une sorte de signifiant flottant.

Dans cet article, il s'agissait d'abord de tracer une généalogie du terme de « Renaissance », et c'est alors vers les conceptions politiques de Cheikh Anta Diop dès les années 1950 qu'on s'est tourné. S'il se revendique socialiste, et si comme le note Pathé Diagne (1997 : 16), « très tôt, il s'était prémuni contre tout mimétisme [...] il se gardera toujours de professer un quelconque antimarxisme », ses références intimes étaient plutôt tournées vers le continent :

66. Décret no 2003-593 du 16 juillet 2003 portant création du Monument de la Renaissance africaine (Journal officiel de la République du Sénégal, nº 6140, 27 décembre 2003).

67. À son arrivé au pouvoir, Macky Sall a lancé une vaste entreprise d'audit des comptes publics, incluant la réalisation du monument. En 2017, cet audit n'avait pas encore débouché sur des conclusions publiques. 
« Dubois, Garvey, Kenyatta ou Padmore» (ibid.). Les lectures de Senghor, sa bibliothèque, sont composées d'ouvrages variés. Au nationalisme droitier de Maurice Barrès, qu'il apprécie pour la place que celui-ci donne à la terre, «le sang lorrain, c'était le sang sérère » dira Senghor (cité dans Vaillant 2006 : 105), bientôt celui-ci complète son apprentissage intellectuel par des auteurs liés à l'Afrique. Dans l'un de ses articles « Vues sur l'Afrique noire ou assimiler, non être assimilés » (Senghor 1964b), ses références principales, et très positives, sont celles de l'administrateur Delavignette ou du général Lyautey. La renaissance de Diop ne pouvait décidément pas être celle de Senghor.

L'archéologie de ces régimes d'historicité panafricains permet alors de se pencher sur ses développements les plus contemporains, même si des enquêtes ultérieures de nature plus socio-anthropologique, auprès de différents segments de la population devraient en préciser les contours. Comme mentionné précédemment, en avril 2007, Abdoulaye Wade fait chanter l'hymne de la Renaissance africaine lors de la cérémonie d'investiture de son deuxième mandat, mais cette même année 2007 marque un nouvel épisode dans la trajectoire de la «Renaissance ». En juillet, le président français en exercice prononce un discours à «l'Université de Dakar». Ne pas nommer le nom de cette institution, comme le remarque Elikia M'Bokolo (2009 : 14), «est significatif ou bien de l'ignorance incroyable du président français et de ses conseillers, ou plutôt d'une volonté délibéré, accompagnée d'une sorte de mépris revanchard, de ne pas citer le nom de celui — Cheikh Anta Diop - [et de son] long et victorieux combat contre les "falsifications de l'histoire" "». Ce discours a déjà été longuement commenté (Ba Konaré 2009; Chrétien et al. 2008), sans qu'il faille y revenir longuement une nouvelle fois. Notons juste que dans ce discours écrit par Henri Guaino, le mot "Renaissance » est prononcé dix fois. De Diop à Sarkozy, en passant par Senghor et Wade, on achève bien les idées. C'est ainsi à une falsification des idéaux et du projet politique de Cheikh Anta Diop que l'on semble avoir assisté, même si probablement le terme de «Renaissance africaine », tout à la fois en tant que mémoire historique et projet politique, «renaîtra » sous d'autres auspices.

Institut historique allemand (IHA); Centre de recherche sur les politiques sociales (CREPOS), Dakar (Sénégal). 


\section{BIBLIOGRAPHIE}

ANDERSON B., 1996, L'imaginaire national. Réflexions sur l'origine et l'essor du nationalisme, Paris, La Découverte.

Arnoldi M. J., 2003, «Symbolically Inscribing the City : Public Monuments in Mali, 1995-2002 », African Arts, XXXVI (2), 95-96 : 56-65.

Assmann J., 2003, Moïse l'Égyptien. Un essai d'histoire de la mémoire, Paris, Flammarion.

BA KonARÉ A. (DIR.), 2009 [2008], Petit précis de remise à niveau sur l'histoire africaine à l'usage du président Sarkozy, Paris, La Découverte.

BASSÈne P. C. B., 2011, Mémoire de l'esclavage et de la traite négrière en Sénégambie (1965-2007). Dialectique de la diversité mémorielle, Thèse de doctorat, Université Laval/Université européenne de Bretagne (Université Bretagne Sud).

Bocoum H. \& Toulier B., 2013, «La fabrication du patrimoine : l'exemple de Gorée (Sénégal) », In Situ, $20: 2-30$.

Boukary-Yabara A., 2014, Africa Unite! Une histoire du panafricanisme, Paris, La Découverte.

CAstoriadis C., 1975, L'institution imaginaire de la société, Paris, Éditions du Seuil.

CÉSAIRE A., 1955, Discours sur le colonialisme, Paris, Présence Africaine.

Chivallon C., 2007, « Retour sur la communauté imaginée d'Anderson. Essai de clarification théorique d'une notion restée floue », Raisons politiques, 27 (3) : 131-172.

Chrétien J-P. et AL., 2008, L'Afrique de Sarkozy. Un déni d'histoire, Paris, Karthala.

Colin R., 2007, Sénégal notre pirogue. Au soleil de la liberté : journal de bord, 1955-1980, Paris, Présence Africaine.

Crouzel I., 2000, «La "Renaissance africaine" : un discours sud-africain ? », Politique Africaine, 77 : 171-182.

De Jong F., 2008, « Recycling Recognition. The Monument as Objet trouvé of the Postcolony », Journal of Material Culture, 13 : 195-214.

De Jong F. \& Foucher V., 2010, «La tragédie du roi Abdoulaye. Néomodernisme et Renaissance africaine dans le Sénégal contemporain », Politique Africaine, 118 : 187-204.

De Jorio R., 2006, « Politics of Remembering and Forgetting : The Struggle over Colonial Monuments in Mali », Africa Today, 52 (4) : 79-105.

Delas D., 2006, Léopold Sédar Senghor. Le maître de la langue, Croissy-Beaubourg, Aden. 
Dewitte P., 1985, Les mouvements nègres en France, 1919-1939, Paris, l'Harmattan.

Diagne P., 1997, Cheikh Anta Diop et l'Afrique dans l'histoire du monde, Paris, L'Harmattan-Sankoré.

— 2002, La Révolution Ramakushi ou l'archéologie linguistique et culturelle de la préhistoire spirituelle et intellectuelle de l'humanité, Dakar, Sankoré.

— 2013, «Abdoulaye Wade ou la fin du cycle senghorien », in M.-C. Diop (dir.), Le Sénégal sous Abdoulaye Wade. Le Sopi à l'épreuve du pouvoir, Paris, Karthala: 97-118.

Diaw A., 1992, «La démocratie des lettrés », in M.-C. Diop (dir.), Sénégal trajectoire d'un État, Dakar, Codesria : 299-329.

Dieng A. A., 2007, «Le $1^{\text {er }}$ Congrès des écrivains et artistes noirs et les étudiants africains », Présence Africaine, 175-176-177 : 118-124.

Diop B. B., 2006, « Le Sénégal entre Cheikh Anta Diop et Senghor », < https://liberalarts. utexas.edu/france-ut/_files/pdf/resources/Diop.pdf $>$.

Diop C. A., 1959, L'unité culturelle de l'Afrique noire. Domaine du patriarcat et du matriarcat dans l'antiquité classique, Paris, Présence Africaine.

— 1974 [1960], Les fondements culturels techniques et industriels d'un futur État fédéral d'Afrique noire, Paris, Présence Africaine.

- 1979 [1954], Nations nègres et culture, Paris, Présence Africaine.

- 1981, Civilisation ou barbarie. Anthropologie sans complaisance, Paris, Présence Africaine.

— 1990, Alertes sous les tropiques. Articles de 1946-1960, Culture et développement en Afrique noire, Paris, Présence Africaine.

Diop C. M., 2003, Cheikh Anta Diop, l'homme et l'œuvre. Aperçu par l'image : les racines du futur, Paris, Présence Africaine.

Diop M.-C., 2013a, Le Sénégal sous Abdoulaye Wade. Le Sopi à l'épreuve du pouvoir, Paris, Karthala.

— 2013b, Sénégal, 2000-2012. Les institutions et politiques publiques à l'épreuve d'une gouvernance libérale, Paris, Karthala.

Diop M.-C. \& Diouf M., 1990, Le Sénégal sous Abdou Diouf, Paris, Karthala.

Diouf M., 1990, Le Kajoor au XIXe siècle. Pouvoir ceddo et conquête coloniale, Paris, Karthala.

— 1992, «Fresques murales et écritures de l'histoire : Le Set/Setal à Dakar », Politique Africaine, $46: 41-54$.

DrAMÉ P., 2011, «La monumentalisation du passé colonial et esclavagiste au Sénégal : controverse et rejet de la renaissance africaine », Revue de la société historique du Canada, 22 : 237-265. 
Fauvelle-Aymard F.-X., 1996, L'Afrique de Cheikh Anta Diop. Histoire et idéologie, Paris, Karthala.

FicQuet É. \& GALLIMARDET L., 2009, «“On ne peut nier longtemps l'art nègre”. Enjeux du colloque et de l'exposition du Premier Festival mondial des arts nègres de Dakar en $1966 »$, Gradhiva, 10 : 134-155.

FoUÉRÉ M.-A., 2010, « La mémoire au prisme du politique », Cahiers d'Études africaines, L (1), $197: 1-16$.

FriouX-SAlgas S., 2009, « Présence Africaine. Une tribune, un mouvement, un réseau », Gradhiva, $10: 5-21$.

Halbwachs M., 1994 [1925], Les cadres sociaux de la mémoire, Paris, Albin Michel.

— 1997 [1950], La mémoire collective, Paris, Albin Michel.

HobSBAWm E. \& RANGER T. (DIR.), 2012 [1983], L'invention de la tradition, Paris, Éditions Amsterdam.

Johnson W., 1991 [1971], Naissance du Sénégal contemporain. Aux origines de la vie politique moderne : 1900-1920, Paris, Karthala.

KIng S., 2015, Whose Harlem is this, anyway? Community Politics and Grassroots Activism during the New Negro Era, New York, New York University Press.

Koselleck R., 2011 [1997], L'expérience de l'histoire, Paris, Points.

ManN G., 2005, «Locating Colonial Histories : between France and West Africa », The American Historical Review, 110 (2) : 409-434.

Mbembe A., 2005 [2000], De la postcolonie. Essai sur l'imagination politique dans l'Afrique contemporaine, Paris, Karthala.

— 2013, Critique de la raison nègre, Paris, La Découverte.

M'Bokolo E., 1996, «Préface », in F. X. Fauvelle-Aymard (dir.), L'Afrique de Cheikh Anta Diop. Histoire et idéologie, Paris, Karthala : 7-13.

— 2009 [2008], «Préface », in A. BA Konaré (dir.), Petit précis de remise à niveau sur l'histoire africaine à l'usage du président Sarkozy, Paris, La Découverte : 9-19.

Michel M., 2013, «René Maran et Blaise Diagne : deux négritudes républicaines », Présence Africaine, 187-188 : 153-166.

Mourre M., 2017a, Thiaroye 1944. Histoire et mémoire d'un massacre colonial, Rennes, Presses universitaires de Rennes.

— 2017b, [à paraître] «"Faire de chaque Sénégalais un Lat-Dior pour vaincre le sousdéveloppement". Invention et actualisation de la figure d'un héros ouest-africain », Cahiers Afrique.

— 2018, [à paraître] «Crime et patrimoine. L'UNESCO, l'île de Gorée et de quelques 
débats sur la traite atlantique », in M. P. Ballarin (dir.), Paris, L'esclavage en Afrique: Histoire, héritage et actualités, Paris, Karthala.

Mudimbe V., 1994, The Idea of Africa, Bloomington, Indiana University Press ; London, James Currey.

Nkrumah K., 2009a [1964], L’Afrique doit s'unir, Paris, Présence Africaine.

— 2009b [1969], Le consciencisme, Paris, Présence Africaine.

RIESZ J., 2011, La création du journal «Condition humaine ». Un tournant dans la vie de Léopold Sédar Senghor, Le Vésinet, Images et mémoires.

SEnghor L. S., 1948, «La Révolution de 1848 et le respect de la personne humaine », Condition humaine : 4.

- 1964a, Liberté I. Négritude et humanisme, Paris, Éditions du Seuil.

- 1964b, «Vues sur l'Afrique noire ou Assimiler, non être assimilés », in Liberté I, Négritude et Humanisme : 39-69.

- 1971, Liberté II, Nation et voie africaine du socialisme, Paris, Éditions du Seuil.

- 1980, La poésie de l'action. Conversations avec Mohamed Aziza, Paris, Stock.

Tine A., 1997, « De l'un au multiple et vice-versa ? Essai sur le multipartisme au Sénégal (1974-1996)», Polis. Revue camerounaise de science politique, 1 (3) : 61-105.

— 2005, «Léopold Senghor et Cheikh Anta Diop face au panafricanisme : deux intellectuels, même combat mais conflit des idéologies ? », in T. BAH (dir.), Intellectuels, nationalisme et idéal panafricain. Perspective historique, Dakar, Codesria : 129-157.

Vaillant J., 2006 [1990], Vie de Léopold Sédar Senghor. Noir, Français et Africain, Paris, Karthala.

Wade A., 1953, «Afrique Noire et Union française », Présence Africaine, 14 : 118-144.

- 1964, Économie de l'Ouest-africain (zone franc). Unité et croissance, Paris, Présence Africaine.

- 1989, Un destin pour l'Afrique, Paris, Karthala.

— 2008, Une vie pour l'Afrique. Entretiens avec Jean-Marc Kalflèche et Delafon Gilles, Neuilly-Sur-Seine, Michel Lafon. 
RÉSUMÉ

Le monument de la Renaissance africaine inauguré en avril 2010 a suscité de nombreux commentaires dans l'espace public comme en sciences sociales. Cet article s'interroge autant sur les ensembles statutaires dans l'espace public dakarois que sur l'idée même de « renaissance» en historicisant ce concept à partir d'une relecture de textes de Léopold Sédar Senghor et de Cheikh Anta Diop, il s'agit d'en montrer les évolutions radicales que ce terme a subi lorsqu'il était utilisé par Abdoulaye Wade à la fin des années 2000.

Mots-clés: Sénégal, Cheikh Anta Diop, Léopold Sédar Senghor, Abdoualye Wade, mémoire collective, monument, Renaissance africaine.

\section{ABSTRACT}

The African Renaissance: from Ideas to the Monument. The Infrastructure of Cheikh Anta Diop, the Culture of Léopold Sédar Senghor and the Sculpture of Abdoulaye Wade. - The Monument of the African Renaissance inaugurated in April 2010 has aroused a great deal of commentary in the public space as well as in the social sciences. This article examines both the statuary in Dakar's public space and the very idea of "renaissance" or "rebirth" by historicizing this concept. With a re-reading of texts by Léopold Sedar Senghor and Cheikh Anta Diop, we wish to show the fundamental shifts this term underwent when it was used by Abdoulaye Wade at the end of the 2000s.

Keywords: Senegal, Cheikh Anta Diop, Leopold Sedar Senghor, Abdoualye Wade, African Renaissance, collective memory, monument. 